\title{
Gains of $13 q$ are correlated with a poor prognosis in liposarcoma
}

\author{
Hannelore Schmidt ${ }^{1}$, Frank Bartel ${ }^{1}$, Matthias Kappler ${ }^{1}$, Peter Würl ${ }^{2}$, Heidemarie Lange ${ }^{1}$, \\ Matthias Bache ${ }^{3}$, Hans-Jürgen Holzhausen ${ }^{1}$ and Helge Taubert ${ }^{1}$ \\ ${ }^{1}$ Institute of Pathology, Martin Luther University Halle-Wittenberg, Halle, Germany; ${ }^{2}$ Surgical Clinic \\ 1, University of Ulm, Ulm, Germany and ${ }^{3}$ Department of Radiotherapy, Martin Luther University \\ Halle-Wittenberg, Halle, Germany
}

\begin{abstract}
Liposarcomas are a phenotypical heterogenous group of tumors divided into four main subtypes: welldifferentiated, dedifferentiated, myxoid/round cell, and pleomorphic. The aim of this study was to compare DNA sequence copy number changes of these subtypes as investigated by comparative genomic hybridization in 36 patients. Comparative genomic hybridization revealed genomic imbalances in tumors of 27 patients (mean 5.6 imbalances per tumor). The most frequent gains were within single regions of 1q, 12q, and 13q. We found a significant correlation of poor overall survival and gain of $13 q 21(P=0.0221), 13 q 22(P=0.0341), 13 q 31$ $(P=0.0410)$, and 13q32 $(P=0.0074)$. The univariate Cox regression analysis revealed an increased risk of tumorrelated death for patients whose liposarcomas possess with gains of 13q21 and 13q32 simultaneously $(P=0.010 ; \mathrm{RR}=7.1 ; 95 \% \mathrm{Cl} 1.6-31.7)$. Furthermore, 12 high-level amplifications were found in tumors of seven patients. In four cases 12q14-q15 and in two cases 13q32-q33 were amplified. We identified in different liposarcoma subtypes characteristic genomic changes: Gains and high-level amplifications of 12q occurred in all 11 investigated well-differentiated liposarcomas, and these changes were often present simultaneously with gains of 1q (mean 5.5 changes). In the two dedifferentiated liposarcomas, gains of $1 q$ in both liposarcomas, and a high-level amplification of $13 q$ were striking. Only eight of the 17 patients with myxoid/round cell liposarcomas showed changes in DNA copy number (mean 3.4 imbalances). In four of these eight cases gains of 13q occurred. The six pleomorphic liposarcomas possessed the most frequent genomic imbalances (mean number 16.3) of all liposarcoma subtypes investigated. These imbalances were in almost all chromosomal regions detected predominantly as over-representations of chromosomes 1, 5p, 13q, and 22q. Summarizing, all subtypes but well-differentiated liposarcomas showed gains of 13q, which were associated with a poor prognosis.

Modern Pathology (2005) 18, 638-644, advance online publication, 12 November 2004; doi:10.1038/modpathol.3800326
\end{abstract}

Keywords: $\mathrm{CGH}$; gain of $13 \mathrm{q}$; liposarcoma subtypes; poor prognosis

Liposarcomas are the most common soft-tissue sarcomas in adults and account for approximately $20 \%$ of all mesenchymal malignancies. The WHO classification divides liposarcomas into four main histological subtypes that are correlated with degree of malignancy: well-differentiated, dedifferentiated, myxoid/round cell, and pleomorphic liposarcomas. ${ }^{1}$ Well-differentiated liposarcomas are tumors of low degree of malignancy. When located in the subcutis, they are usually cured by local surgical resection and rarely recur. ${ }^{2}$ Comprising $40-45 \%$ of all lipo-

Correspondence: Dr H Schmidt, PhD, Institute of Pathology, Faculty of Medicine, Martin Luther University Halle-Wittenberg, Magdeburger Strasse 14, D-06097 Halle, Germany.

E-mail: hannelore.schmidt@medizin.uni-halle.de

Received 22 June 2004; revised and accepted 21 September 2004; published online 12 November 2004 sarcomas, the well-differentiated liposarcomas are thus the largest subgroup of adipocytic malignancies. Cytogenetic characteristics of well-differentiated liposarcomas are rings as well as giant marker chromosomes that contain amplified genetic material derived from chromosome region 12q13q15 and, alternatively, additional material from chromosomes 1,4 , and $16 .^{3-6}$ Dedifferentiated liposarcoma is a high-grade sarcoma that arises in patients with pre-existing well-differentiated liposarcoma $^{7}$ and shows the same cytogenetic aberrations as well-differentiated liposarcoma. Myxoid/ round cell liposarcoma account for about $30 \%$ of all liposarcomas and share the typical reciprocal translocations $\mathrm{t}(12 ; 16)(\mathrm{q} 13 ; \mathrm{p} 11)$ and $\mathrm{t}(12 ; 22)(\mathrm{q} 13 ; \mathrm{q} 12)$, and trisomy 8 , isochromosome $7 \mathrm{q}$, and $6 \mathrm{q}$ deletions as secondary aberrations. ${ }^{4}$ Pleomorphic liposarcoma is defined as a high-grade liposarcoma and is 
associated with a very poor prognosis. ${ }^{8}$ Complex chromosomal changes without specific rearrangements have been detected in karyotypic analyses of pleomorphic liposarcomas. ${ }^{4}$

Investigations of liposarcomas by comparative genomic hybridization (CGH) have shown a broad scattered pattern of genomic imbalances. ${ }^{9-11}$ In this study, we analyzed altogether 36 cases of four liposarcoma subtypes by CGH to identify distinct DNA sequence copy number changes in the different subtypes and to evaluate their prognostic relevance.

\section{Materials and methods}

\section{Tumor Specimens}

The investigated tumor material consisted of 36 liposarcomas (11 well-differentiated, two dedifferentiated, 17 myxoid/round cell, and six pleomorphic liposarcomas) from 36 patients (17 women, 19 men). The samples were collected from the Institute of Pathology at the Martin Luther University Halle-Wittenberg and from the Surgical Clinic 1 of the University of Leipzig (Germany) within a period of 15 years. We analyzed 27 primary tumors, eight recurrences, and one metastasis with histological grades 1 (16 cases), 2 (16 cases), and 3 (four cases), according to the Van Unnik grading system. ${ }^{12}$ The material investigated consisted of 15 tumors with stage I, 12 tumors with stage II, four tumors with stage III, and five tumors with stage IV (UICC guidelines ${ }^{13}$ ). The 27 primary tumors were untreated before resection, of the eight recurrences only two patients (cases 20 and 30) received radiotherapy after primary tumor resection, and the one patient with metastasis (case 28) was treated by chemotherapy after primary tumor resection. In 20 cases, the liposarcomas were located in the lower extremity, in two cases in the upper extremity, in six cases in the external pelvis, in four cases in the retroperitoneum, in two cases in the thorax, and in two cases were found at multiple locations. The average age at diagnosis was 51 years (range 24-79). Of the 36 patients, 11 died from the disease and 25 are alive. The average survival time was 34 months (11-78 months), and the average observation time for patients who survived was 42 months (6-98 months).

\section{CGH Analysis}

Tumor DNA was isolated from 22 frozen samples and 14 paraffin-embedded samples. The reference DNA was isolated from the blood of healthy probands. CGH was performed as described by Kallioniemi et $a l^{14}$ but with some previously reported modifications. ${ }^{15}$ Briefly, tumor DNA was labeled with biotin-16-dUTP, and reference DNA was labeled with digoxigenin-11-dUTP (Boehringer Mannheim, Mannheim, Germany) by using nick translation. Hybridization was performed for 3 days at $37^{\circ} \mathrm{C}$. The biotinylated sequences were detected with avidin-fluorescein isothiocyanate (FITC) (Boehringer Mannheim), and probe sequences haptenized with digoxigenin were visualized with antidigoxigenin rhodamine (Boehringer Mannheim). Chromosomes were counterstained with DAPI (4,6-diamino-2-phenylindole) (Serva, Heidelberg, Germany).

\section{Analysis of Digital Images Resulting from CGH}

The hybridization was analyzed by the ISIS digital image analysis system (MetaSystems, Altlussheim, Germany). ${ }^{15}$ Chromosomal regions with a green to red ratio above 1.25 were considered to be overrepresented (gains), whereas regions with a ratio below 0.75 were considered to be under-represented (losses). The cutoff value for high-level amplifications was 1.5 according to Kallioniemi et al. ${ }^{14}$ The results were confirmed by calculating the double standard deviation of the individual ratio profiles for each chromosome within the same experiment. Heterochromatic regions were excluded from analysis.

\section{Statistical Analysis}

We used SPSS 11.0 software (SPSS Inc., Chicago, IL, USA) to perform statistical analyses, which included the $\chi^{2}$-test, the Kaplan-Meier method, the univariate Cox regression analysis, and the log-rank test to determine statistical significance. The level of significance was set at $P<0.05$.

\section{Results}

\section{CGH Analysis}

Genomic imbalances were detected in tumors from 27 of the 36 investigated patients with a mean value of 5.6 imbalances per tumor (range 1-23). The DNA sequence copy number changes are given in Table 1. The chromosomal regions that were most frequently affected are shown in Figure 1.

The minimal common regions of the most frequent gains in the 36 liposarcomas were 12q14-q15 (15 cases); 12q21 (13 cases); 12q13 (12 cases); 1q23 and 12q22 (11 cases each); 1q21-q22 and 1q24-q25 (10 cases each); 1q31 and 13q31 (nine cases each); $13 q 22$ and 13q32 (eight cases each). A total of 12 high-level amplifications were observed in tumors of seven patients. The region 12q14-q15 was amplified in four samples, and the region 13q32q33 in two samples. The other high-level amplifications occur once.

Losses were detected strikingly less frequently than gains. Only the chromosomal region 16q12.1qter was characterized by losses in three cases, the other regions of losses occurred only once or twice. 
Table 1 Copy number changes in 36 liposarcomas analyzed by CGH

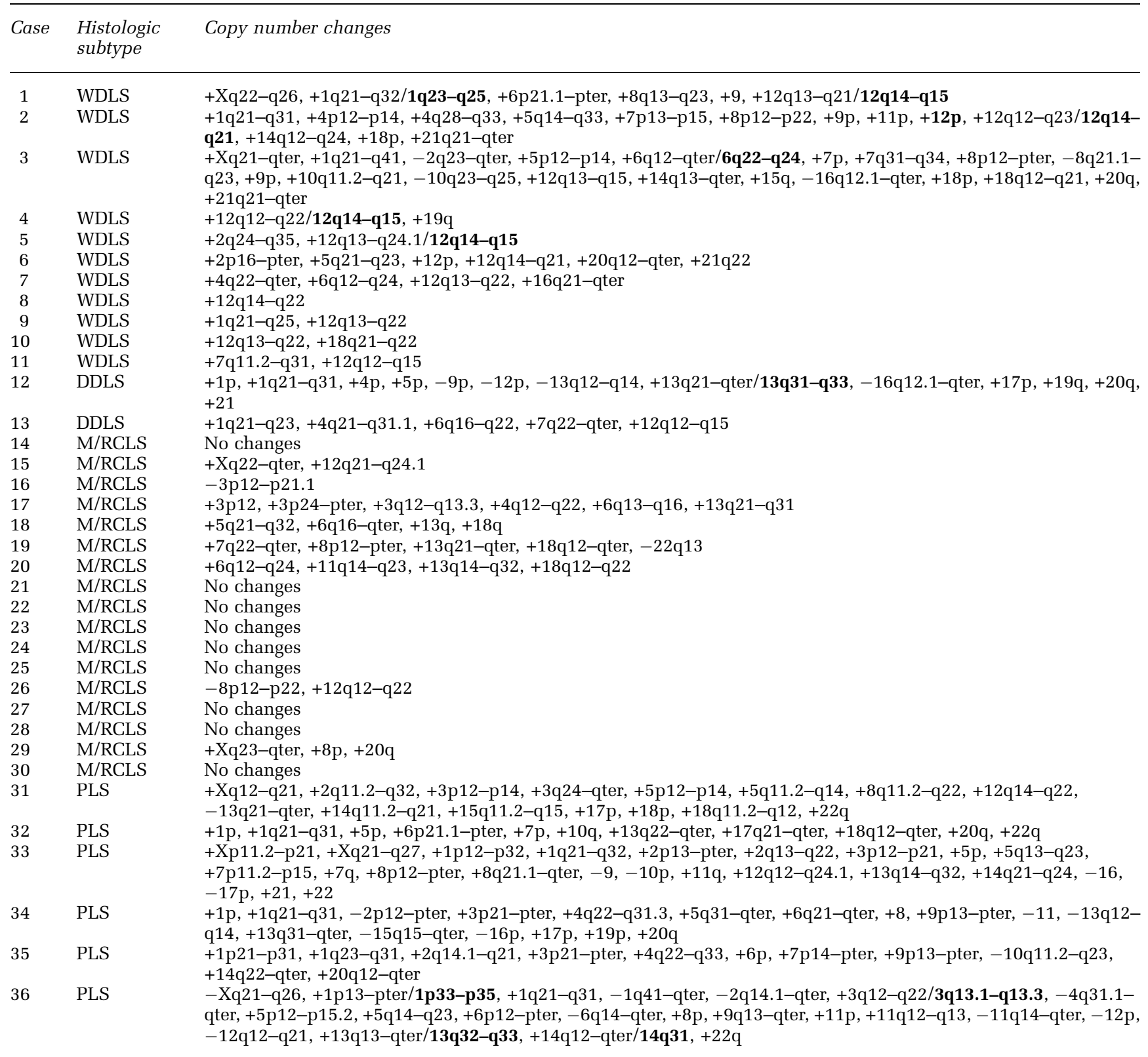

${ }^{a}$ WDLS, well-differentiated liposarcoma; DDLS, dedifferentiated liposarcoma; M/RCLS, myxoid/round cell liposarcoma; PLS, pleomorphic liposarcoma.

Gains of DNA sequences are marked with +, losses with -; high-level amplifications are indicated in bold.

\section{Correlations between Frequent Genomic Imbalances, Clinicopathological Parameters and Overall Survival}

A significant correlation of poor overall survival and gain of 13q21 $(P=0.0221), 13 q 22(P=0.0341), 13 q 31$ $(P=0.0410)$, and 13q32 $(P=0.0074)$ were found in Kaplan-Meier analysis. The patients without gains of $13 \mathrm{q}$ had a mean survival time of 78 months, whereas the patients with gains of $13 q$ survived on average 35 months. Frequent gain of single regions of chromosomes 1q, 12q, and 20q (and also of 2q, 3p, $5,6 p, 7 p, 8,14 q$, and $22 q$ in pleomorphic liposarco- mas detected) did not have any significant influence on patient prognosis (data not shown). In addition, we observed a prognostic impact on overall survival of tumor stage $(P<0.00001)$ and tumor grade $(P=0.0341)$. Tumor subtype and age of patients did not have any significant effect on patient prognosis.

The univariate Cox regression analysis revealed an increased risk of tumor-related death for patients with gains of $13 \mathrm{q} 21(P=0.037 ; \mathrm{RR}=4.2 ; 95 \% \mathrm{CI}$ $1.1-16.4)$, gains of $13 q 32(P=0.020 ; R R=6.0 ; 95 \%$ CI 1.3-27.1), and for patients whose liposarcomas 

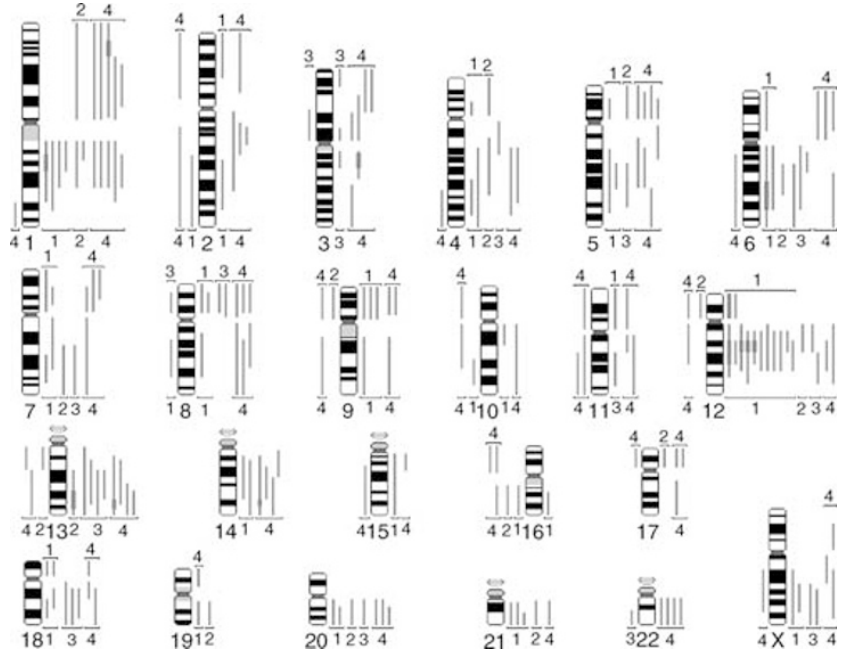

Figure 1 Summary of gains and losses found by CGH in liposarcomas from 36 patients. Losses are shown to the left and gains to the right. High-level amplifications are shown as thick lines. Clamp 1, well-differentiated liposarcomas; clamp 2, dedifferentiated liposarcomas; clamp 3, myxoid/round cell liposarcomas; clamp 4, pleomorphic liposarcomas.

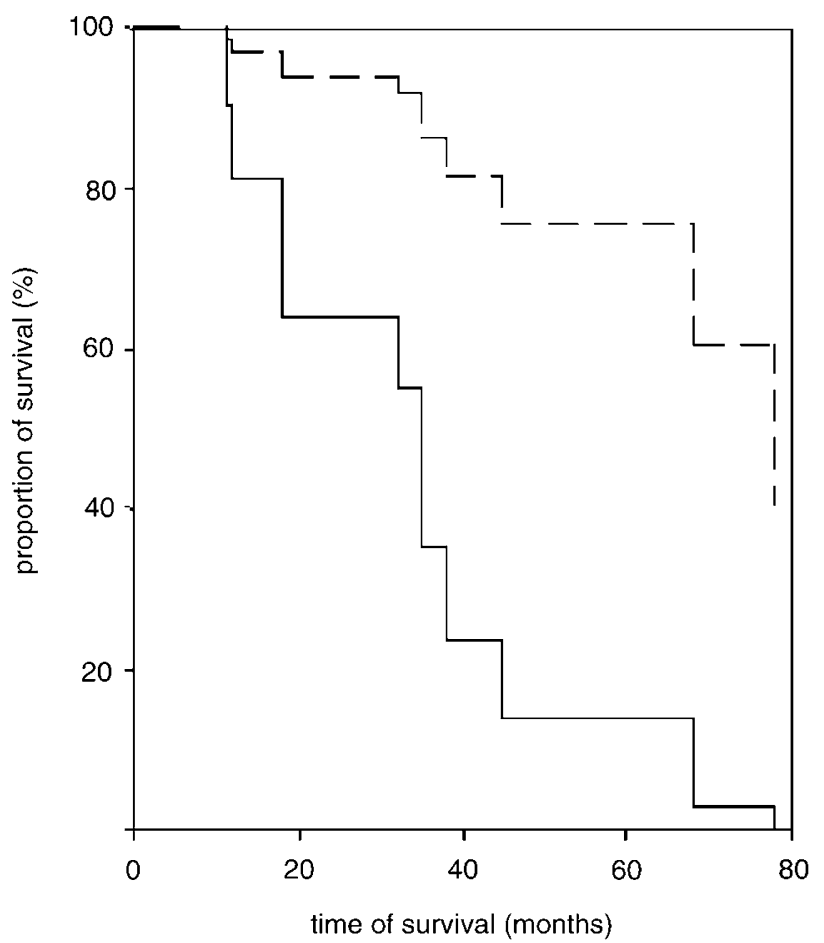

Figure 2 Univariate Cox regression analysis shows a significant correlation between survival of liposarcoma patients and simultaneous gain of 13q21 and 13q32 in their tumors. The broken line represents patients with tumors without gains of 13q, and the continuous line represents those with gains of $13 q(P=0.010$; $\mathrm{RR}=7.1$ ).

possess with both gains of $13 \mathrm{q}(P=0.010$; $\mathrm{RR}=7.1$; 95\% CI 1.6-31.7) (Figure 2).

To investigate the correlation of prognostic relevant gains of $13 \mathrm{q}$ and the different groups of tumor
Table 2 Occurrence of gains at single regions of $13 q$ in tumors with different subtypes ( $\chi^{2}$-test)

\begin{tabular}{|c|c|c|c|c|c|}
\hline & $\begin{array}{c}\text { Total number of } \\
\text { cases }\end{array}$ & $+13 q 21$ & $+13 q 22$ & $+13 q 31$ & $+13 q 32$ \\
\hline WDLS & 11 & 0 & 0 & 0 & 0 \\
\hline DDLS & 2 & 1 & 1 & 1 & 1 \\
\hline M/RCLS & 17 & 4 & 4 & 4 & 3 \\
\hline PLS & 6 & 2 & 3 & 4 & 4 \\
\hline$P$-value & & 0.190 & 0.081 & 0.019 & 0.011 \\
\hline
\end{tabular}

WDLS, well-differentiated liposarcoma; DDLS, dedifferentiated liposarcoma; M/RCLS, myxoid/round cell liposarcoma; PLS, pleomorphic liposarcoma.

stages, tumor grades, and tumor subtypes we used the $\chi^{2}$-test. There were positive correlations of gains at $13 \mathrm{q} 31$ and $13 \mathrm{q} 32$ with higher tumor stage $(P=0.035$ and 0.008), higher tumor grade $(P=0.013$ and 0.003$)$, and pleomorphic liposarcoma $(P=0.019$ and 0.011$)$. Table 2 shows the distribution of cases with gains of the single regions of $13 q$ in the tumors with different subtypes.

\section{Frequent Genomic Imbalances in Different Subtypes of Liposarcomas}

\section{Well-differentiated and dedifferentiated liposarcomas}

The mean number of genomic imbalances in 11 well-differentiated liposarcomas was 5.5 (range 1-20). All well-differentiated liposarcomas showed gains of chromosome arm 12q: 12q13 (nine cases), 12q14-q15 (11 cases), 12q21 (10 cases), and 12q22 (eight cases). We could observe seven high-level amplifications in five well-differentiated liposarcomas. The four high-level amplifications at 12q14q15 (one of which extended to 12q21) occurred only in well-differentiated liposarcomas. Furthermore, one high-level amplification at 1q23-q25, one at 6q22-q24, and another at 12p were detected. Losses were found only in two cases (Figure 1, clamps 1; Table 1).

One (case 13) of the two dedifferentiated liposarcomas possessed five regions of gain. In the other tumor (case 12), nine regions with gain, one highlevel amplification at 13q31-q33, and four regions with loss were detected. Only a gain of 1q21-q23 was observed in both samples of dedifferentiated liposarcomas (Figure 1, clamps 2; Table 1).

\section{Myxoid/round cell liposarcomas}

Genomic imbalances were observed less frequently in myxoid/round cell liposarcomas than in other subtypes of liposarcoma. Only eight of the 17 myxoid/round cell liposarcomas showed changes in DNA copy number (mean 3.4 imbalances per tumor; range 1-6). Gains were identified most frequently in 13q21-q31 (four cases); 6q16, 13q32, 
and 18q12-q22 (three cases each). No high-level amplifications, and only one loss each were found in three myxoid/round cell liposarcomas (Figure 1, clamps 3; Table 1).

\section{Pleomorphic liposarcomas}

In the six pleomorphic liposarcomas, the mean number of genomic imbalances was 16.3 (range 11-23). Changes occurred in all chromosomes. We found in all six pleomorphic liposarcomas gains, mostly in the regions 1p21-p31, and 1q23-q31 (five cases each), 1p13, 1p32, 1q21-q22, 5p12-p14, 13q31-q32, and 22q (four cases each). In three of the six pleomorphic liposarcomas, gains were limited to the regions 1p12, 1p33-pter, 2q14.1q21, 3p21, 5p15.1-p15.2, 5q14, 6p21.1-pter, 7p14p15, 8p12-pter, 8q21.1-q22, 13q22, 13q33-qter, 14q21-q24, and 20q12-qter. One pleomorphic liposarcoma contained four high-level amplifications at 1p33-p35, 3q13.1-q13.3, 13q32-q33, and $14 q 31$. Losses were observed in five pleomorphic liposarcomas (mean 3, range 1-8) (Figure 1, clamps 4; Table 1).

\section{Discussion}

The aim of this study was the comparison of genomic imbalances in the four main subtypes of liposarcoma investigated by CGH. The most frequent DNA copy number changes were found as gains of single chromosomal regions of 1q, 12q, and 13q. The number of detected losses is very low. Whereas gains of $1 \mathrm{q}$ and $12 \mathrm{q}$ were reported previously,,$^{3,9-11,16-}$ ${ }_{18}$ the frequent gain of $13 \mathrm{q}$ is not reported in the 111 liposarcomas investigated by CGH until now. The present study showed that gains of $13 q$ are correlated significantly with a poor prognosis, and this finding has been described in liposarcoma subtypes for the first time. Comparable findings of copy number gains of single regions of $13 q$ has been reported also in other sarcomas, for example, in malignant fibrous histiocytomas, ${ }^{19}$ and alveolar rhabdomyosarcomas. ${ }^{20}$ However, these authors could not find any prognostic relevance of gains of $13 q$ regions. Using a univariate Cox regression analysis we showed that the patients whose liposarcomas possess with gains at 13q, have an increased risk of tumor-related death. We found a significant positive correlation of gains at $13 q 31$ and 13 q32 with higher tumor grades and tumor stages. About relevant target genes we can only speculate. The pancreatic cancer-derived gene PCD1 (13q21.33) is highly expressed in colon, breast, liver, lung, pancreas, stomach, and prostate tumor tissues. $^{21}$ However, with RT-PCR we could not detect an overexpression of PCD1 in liposarcomas with a gain of $13 q$ (data not shown).

The copy number changes in the analyzed 36 liposarcomas varied widely, but the single subtypes of this tumor group have characteristic genomic differences.

In the 11 investigated well-differentiated liposarcomas the most frequent gain was that of 12q; we also found four high-level amplifications of 12q14q15. These high-level amplifications were found exclusively in well-differentiated liposarcomas. Four tumors with gains of $12 q$ also had gains of 1q. This coamplification of both regions of $1 \mathrm{q}$ and $12 q$ was characteristic for well-differentiated liposarcomas. As target genes in the region of $12 \mathrm{q}$, for example, the amplicons SAS, CDK4 (12q13-q14), and MDM2 (12q14.3-q15) are known in sarcomas. Previously, we have investigated nine of the 11 welldifferentiated liposarcomas of the present study by semiquantitative PCR for MDM2 amplification. ${ }^{22}$ Of the nine cases, we found in four cases both highlevel amplifications of 12q14-q15 by CGH analyses and an amplification of MDM2 by semiquantitative PCR. ${ }^{22}$ In four out of the other five cases, gains of 12q13-q15 were detected, which carried in two cases an MDM2 amplification as well. At the time this paper was written, 41 well-differentiated liposarcomas had been analyzed by CGH. Gains, highlevel amplifications, or both, of subregions of $1 \mathrm{q}$ and $12 \mathrm{q}$ are the imbalances that have been described most frequently. ${ }^{2,3,9,10,16,17}$ The results of our analyses of 11 well-differentiated liposarcomas confirmed the findings of these earlier reports.

In the two analyzed dedifferentiated liposarcomas, the remarkable findings were the over-representation of 1q regions in both cases and a high-level amplification of 13q. Other CGH analyses revealed over-representation of single regions of $12 q$ in each of 30 cases studied to date ${ }^{10,11,17,18}$ and frequent gains of $6 q$ regions and $20 q 13 .{ }^{11}$ In our study a gain of 6q16-q22, 12q12-q15, and 20q was identified in one case each.

In nine of 17 myxoid/round cell liposarcomas we detected no genomic imbalance. In the other eight tumors, the most frequent change was a gain of subregions of 13q (four cases). We detected neither gains of 1q nor high-level amplifications of 12q as in well-differentiated liposarcomas. The absence of any high-level amplification was characteristic for myxoid/round cell liposarcoma. Other CGH analyses of 16 cases of myxoid/round cell liposarcomas showed over-representation of chromosome 8 as the most frequent aberration. ${ }^{3,9,17}$ However, our analysis of $17 \mathrm{myxoid} /$ round cell liposarcomas revealed an over-representation of 8p12-pter in only two cases. Altogether we suggest that the development of myxoid/round cell liposarcomas is characterized more by structural aberrations that occur at the chromosome level but do not disrupt the genomic balance.

Our CGH analyses of six pleomorphic liposarcomas revealed genomic imbalances in all chromosomes with the highest mean aberration number of 16.3 per tumor. These results confirmed ealier findings of 24 pleomorphic liposarcomas 
analyzed. 9,11,16,17 Most frequently, we found DNA sequence copy number gains of regions of the chromosomes $1,5 p, 13 q$, and $22 q$, also described in other sarcomas. ${ }^{23}$ In the CGH analysis described by Rieker et $a{ }^{11}{ }^{11}$ the gain of $20 q 13$ was the most frequent genomic imbalance in pleomorphic liposarcomas. In the present study, in half of the pleomorphic liposarcoma samples, a gain of 20q12-qter was detected by CGH as well.

In conclusion, we have shown that there are a few distinct cytogenetic differences between subtypes of liposarcoma. In general, the pleomorphic liposarcomas possessed the most frequent genomic imbalances, which occurred in almost all chromosomal regions. However, pleomorphic liposarcomas can be distinguished from well-differentiated liposarcomas and also from dedifferentiated liposarcomas by the lack of high-level amplifications in 12q13-q15, the loci of $S A S, C D K 4$, and MDM2. These oncogenes are amplified exclusively at a high level in welldifferentiated and dedifferentiated liposarcomas. However, well-differentiated liposarcomas differ from dedifferentiated liposarcomas by the absence of detectable gains and high-level amplifications of 13q. It is intriguing that a gain of $13 q 21-q 32$ is correlated significantly with a poor prognosis in different liposarcoma subtypes. The myxoid/round cell liposarcomas generally lacked frequent or striking genomic imbalances detected by CGH analyses. We observed no gains of $1 \mathrm{q}$ and no highlevel amplifications of 12q. Predominantly, myxoid/ round cell liposarcomas were characterized by the known reciprocal translocations.

\section{Acknowledgements}

We thank Mrs B Leuschner and Mrs I Wiederhold for their excellent technical assistance; Mrs C BurnsKlein's work in revising the translation of the manuscript is especially appreciated. We are very thankful for the editing of the manuscript by Dr Julia Cay Jones. This work was supported by MildredScheel-Stiftung 'Deutsche Krebshilfe'; Grant numbers: 10-1728 and 10-2130-Ta2.

\section{References}

1 Fletcher CDM, Unni KK, Mertens F (eds). Pathology and Genetics of Tumours of Soft Tissue and Bone. IARC Press: Lyon, 2002.

2 Forus A, Larramendy ML, Meza-Zepeda LA, et al. Dedifferentiation of a well-differentiated liposarcoma to a highly malignant metastatic osteosarcoma: amplification of 12q14 at all stages and gain of 1q22-q24 associated with metastases. Cancer Genet Cytogenet 2001a;125:100-111.

3 Suijkerbuijk RF, Olde Weghuis DEM, van den Berg M, et al. Comparative genomic hybridization as a tool to define two distinct chromosome 12-derived amplifica- tion units in well-differentiated liposarcomas. Genes Chromosomes Cancer 1994;9:292-295.

4 Sreekantaiah C. The cytogenetic and molecular characterization of benign and malignant soft tissue tumors. Cytogenet Cell Genet 1998;82:13-29.

5 Pedeutour F, Forus A, Coindre J-M, et al. Structure of the supernumerary ring and giant rod chromosomes in adipose tissue tumors. Genes Chromosomes Cancer 1999;24:30-41.

6 Forus A, Bjerkehagen B, Sirvent N, et al. A welldifferentiated liposarcoma with a new type of chromosome 12-derived markers. Cancer Genet Cytogenet 2001b;131:13-18.

7 Nascimento AG. Dedifferentiated liposarcoma. Semin Diagn Pathol 2001;18:263-266.

8 Oliveira AM, Nascimento AG. Pleomorphic liposarcoma. Semin Diagn Pathol 2001;18:274-285.

9 Parente F, Grosgeorge J, Coindre JM, et al. Comparative genomic hybridization reveals novel chromosome deletions in 90 primary soft tissue tumors. Cancer Genet Cytogenet 1999;115:89-95.

10 Micci F, Teixeira MR, Bjerkehagen B, et al. Characterization of supernumerary rings and giant marker chromosomes in well-differentiated lipomatous tumors by a combination of G-banding, CGH, M-FISH, and chromosome- and locus-specific FISH. Cytogenet Genome Res 2002;97:13-19.

11 Rieker RJ, Joos S, Bartsch C, et al. Distinct chromosomal imbalances in pleomorphic and in high-grade dedifferentiated liposarcomas. Int J Cancer 2002;99: 68-73.

12 Unnik van JAM, Coindre JM, Contesso G, et al. Grading of soft tissue sarcomas. In: Ryan JR, Baker LO (eds). Experience of the EORTC Soft-tissue and Bone Sarcoma Treatment. Kluwer: Dordrecht, 1988, pp 7-13.

13 Wittekind C, Wagner G (eds). UICC - TNM-Klassifikation maligner Tumoren. Springer: Berlin, 1997.

14 Kallioniemi O-P, Kallioniemi A, Piper J, et al. Optimizing comparative genomic hybridization for analysis of DNA sequence copy number changes in solid tumors. Genes Chromosomes Cancer 1994;10:231-243.

15 Schmidt $\mathrm{H}$, Würl $\mathrm{P}$, Taubert $\mathrm{H}$, et al. Genomic imbalances of $7 p$ and $17 q$ in malignant peripheral nerve sheath tumors are clinically relevant. Genes Chromosomes Cancer 1999;25:205-211.

16 Forus A, Weghuis DO, Smeets D, et al. Comparative genomic hybridization analysis of human sarcomas: I. Occurrence of genomic imbalances and identification of a novel major amplicon at 1q21-q22 in soft tissue sarcomas. Genes Chromosomes Cancer 1995;14: 8-14.

17 Szymanska J, Tarkkanen M, Wiklund T, et al. Gains and losses of DNA sequences in liposarcomas evaluated by comparative genomic hybridization. Genes Chromosomes Cancer 1996;15:89-94.

18 Hostein I, Coindre JM, Derre J, et al. Comparative genomic hybridization study of paraffin-embedded dedifferentiated liposarcoma fixed with Holland Bouin's fluid. Diagn Mol Pathol 2003;12:166-173.

19 Larramendy ML, Tarkkanen M, Blomqvist C, et al. Comparative genomic hybridization of malignant fibrous histiocytoma reveals a novel prognostic marker. Am J Pathol 1997;15:1153-1161.

20 Gordon AT, Brinkschmidt C, Anderson J, et al. A novel and consistent amplicon at 13q31 associated with alveolar rhabdomyosarcoma. Genes Chromosomes Cancer 2000;28:220-226. 
21 Kang S, Xu H, Duan X, et al. PCD1, a novel gene containing PDZ and LIM domains, is overexpressed in several human cancers. Cancer Res 2000;60:5296-5302.

22 Bartel F, Meye A, Würl P, et al. Amplification of the MDM2 gene, but not expression of splice variants of
MDM2 mRNA, is associated with prognosis in soft tissue sarcoma. Int J Cancer 2001;95:168-175.

23 Schmidt H, Hinze R. Zytogenetik. In: Rath F-W, Schönfelder M (eds). Weichteilsarkome des Erwachsenen. Karger: Freiburg, 2002, pp 52-66. 